\title{
A re-examination of matrix effects in the segmented-flow analysis of nutrients in sea and estuarine water
}

\author{
Stephen Coverly ${ }^{a,}{ }^{*}$, Roger Kérouel ${ }^{b}$, Alain Aminot $^{b}$ \\ a SEAL Analytical GmbH, Werkstrasse 5, 22844 Norderstedt, Germany \\ ${ }^{b}$ IFREMER-Brest, BP 70, 29280 Plouzané, France \\ *: Corresponding author : Stephen Coverly, Tel. +49 40609292911 ; email address : \\ Stephen.Coverly@seal-analytical.com ; Roger Kérouel, email address : Roger.Kerouel@ifremer.fr
}

\begin{abstract}
:
Modern instruments together with the use of standard reference materials have improved the accuracy and long-term reproducibility of the analysis of nutrients (ammonium, nitrate, nitrite, phosphate and silicate) in sea water using segmented-flow analysis, so that errors arising from matrix effects become more significant. Colorimetric detectors with bubble-through flowcells have become widely used for seawater analysis in recent years and their associated matrix effects are described. A re-examination of all categories of matrix effects, whose main origin is salinity, was thus undertaken to assess how much they are liable to alter the data. Interferences were classified into four types, each of which was examined in order to show its influence on the measurements. We discuss how matrix effects can be identified, quantified, reduced and corrected. Chemistry and instrument design play a major role in some matrix effects and recommendations are given to minimise these or make them easier to correct. In particular, the correction for chemical salt effects is revisited for the general case and new, simplified procedures are proposed for its computation. A semi-automated procedure is proposed for measuring and correcting sample and refractive index blanks.
\end{abstract}

\section{Highlights}

We reclassify matrix effects and distinguish between static and dynamic origins. We contrast matrix effects in debubbled and bubble-through flowcells. We show how matrix effects can be measured and reduced. We propose simplified correction procedures for chemistry-related matrix effects. We propose a semi-automated correction procedure for refractive index blanks.

Keywords : Nutrient ; Determination ; Seawater ; Estuarine water ; Segmented-flow analysis ; Matrix effects 


\section{Introduction}

Fifty-three laboratories from eighteen countries took part in the 2006 inter-laboratory Comparison Study for Reference Material for Nutrients in Seawater (RMNS) [1], whose objective is to improve reproducibility between laboratories by using identical calibration reference standards. During a subsequent workshop, which was the origin of this paper, attention was paid to other sources of error in nutrient analysis of which one is matrix effects. In seawater nutrient analysis, variations in matrix composition are known to alter the results of some measurements, and some analytical instruments generate matrix-related peak artefacts.

Several decades ago the analysis of nutrients in seawater gave rise to relatively standard colorimetric methods more or less affected by salinity, the so-called "salt effect", and with the development of automated flow analysis instruments some authors paid attention to optical matrix effects generated by the instrument itself, in particular those altering the measured concentrations and the refractive index blank [2-5].

It is clear that matrix effects are most relevant to the analysis of seawater samples of varying salinity, where considerable errors can occur if the method sensitivity is strongly affected by salt content [3]. In contrast, measurements in open ocean samples of constant salinity are generally assumed to be free of matrix effects that alter results provided standards and baseline are prepared in water having the same composition as the samples. However, little attention has been paid to the influence of the solution used for inter-sample wash in a segmented-flow analyzer with a bubble-through flowcell; this can create optical perturbations that alter results in ways not previously described.

More than $90 \%$ of the participants in [1] used a segmented-flow analyer; this technology is generally preferred for seawater nutrient analysis because the lower inter-sample dispersion in a bubble-segmented stream helps to separate the sample from the inter-sample wash solution; most dynamic matrix effectss occur at this interface and it is advantageous if it passes quickly through the flowcell. Furthermore, extended steady-state periods at maximum sample concentration are easily achieved in a segmented-flow system, and this contributes to low detection limit. Accordingly, this paper considers only segmented-flow analyzers and we pay special attention to matrix effects in bubble-through flowcells, which were used by ten of the twelve laboratories in [1] with the lowest Z-scores.

In this paper we propose a compilation of the sources of matrix effects according to a rational classification not yet described in the literature. We discuss how to identify, quantify, reduce and correct different kinds of matrix effects. Experimental results illustrate these effects and show how reaction conditions, choice of methodology or the characteristics of the detector may influence matrix effects. We also propose simpler ways to correct them if this is required by the expected analytical precision.

\section{Experimental}

The SFA systems used in this experimental work are Bran + Luebbe AutoAnalyzer 2, Bran + Luebbe AutoAnalyzer 3 and Seal Analytical AA3 HR. Unless otherwise stated the methods are as described by Aminot et al. [7, 12]. The methods used for the work in this paper are those for ammonium and phosphate. For ammonium the colorimetric phenol-hypochlorite method and the OPA-fluorometric methods were compared. For phosphate, the phosphomolybdenum blue colorimetric method automates Murphy and Riley's procedure [17] as closely as possible. A summary of these methods is given in Table 1. Samples were free of suspended matter. 


\section{Results and discussion}

\subsection{Matrix effect description and classification}

The salinity and other properties of the sample affect the results from a SFA system in ways which depend on the reaction kinetics and the analytical instrument design. Loder and Glibert [2] defined four influences on the absorbance peak obtained by a continuous flow analyzer for dissolved micronutrients in sea water and estuarine samples: the refractive index (RI) of the samples and the liquid used for the sampler wash; reaction and precipitation products of wetting agents and sea water; the absorbance of particulates or dissolved coloured substances; and shifts in the equilibrium of the colour reaction depending on the ionic composition of the sample. We now distinguish between static and dynamic refractive index interference and have reclassified matrix effects as being due to:

1) Sample-specific and chemistry-related effects

a) Matrix absorbance, scattering or fluorescence

b) Salt influence on the reaction rate or end point

2) Hardware-related effects

a) Static salt influences on the optical signal, such as the refractive index blank (RIB)

b) Dynamic salt influences on the optical signal, such as schlieren and lens effects.

\subsection{Sample-specific and chemistry-related effects}

These matrix effects vary from sample to sample and are specific to a particular analytical method. They are due to one or more of three factors: matrix absorbance, scattering and fluorescence.

a) Matrix absorbance may occur in coastal and estuarine waters containing humic acids or any compound that colours the water. The degree of interference depends on the wavelength of measurement and therefore the method in use. Light scattering due to particulates such as those from soil erosion or phytoplankton cells affects all methods: it can be eliminated by filtering or centrifuging the sample before analysis. Fluorescence is unlikely but might occur in estuarine samples or river plumes contaminated by industrial waste.

b) Salt effects on the reaction rate or end point are observed when one or several components in sea water interfere with the reaction between the nutrients in the sample and the colour reagents. This may result in variable formation of reaction products or a shift in the position of equilibrium in the final reaction. For example, in the indophenol blue spectrophotometric method for ammonia a salt effect is generated by incomplete chelation of magnesium ions and a variable buffer effect as a function of salinity, which alter the final $\mathrm{pH}$ of the reaction solution. The literature has described the way in which sample salinity variation affects the results observed in both manual and automated methods [2-6]. The wetting agent may also react with components of the sample or reagents. For example, Levor IV may precipitate and generate an interfering turbidity if its concentration exceeds a given value depending on reaction conditions such as salinity and $\mathrm{pH}$ [2]. It is now largely replaced by high purity sodium 
dodecyl sulphate in methods for phosphate and silicate analysis. Levor IV also reacts with the amine reagent in the determination of nitrite, where it has been replaced by Brij 35 [5].

\subsection{Hardware-Specific Effects}

\subsubsection{Static effects on the optical signal}

Flowcell geometry can affect the measured value of samples whose absorbance is the same but whose refractive index varies, for example due to the presence of sea salts. Ideally, light should pass through the flowcell as a parallel beam. In reality it is deflected to a degree which is affected by the angle at which light enters the flowcell, the smoothness and curvature of the flowcell face where the light enters and leaves, and internal absorption, reflection and refraction from the flowcell walls $[6,7]$. Thus in practice the light deviates on its passage through the cell. This occurs whether the liquid is moving or stationary. The light deviation varies according to the refractive index of the liquid and it gives rise to apparent absorbance changes even in the absence of colour-producing reagents. It is thus specific to the detector and depends on the flow cell design and material, light source and optics as well as the refractive index. This apparent absorbance has been described as the refractive index correction [2] or preferably the refractive index blank (RIB). Usually, when the refractive index of the sample is larger than that of the wash, a small apparent increase of absorbance occurs and thus a positive RIB is measured. However, occasionally a negative RIB occurs; this is attributed to a cell geometry which better focuses the beam onto the detector when the sample (as opposed to the inter-sample wash liquid) is in the cell.

\subsubsection{Dynamic salt effects on the optical signal}

Lens effects caused by varying refractive index in a moving stream of warm air were described by Hooke in 1665 [8] and are sometimes termed schlieren, from the German word meaning striations. Similar effects occur in liquid masses or streams when the refractive index changes due to the mixing of warm and cold zones or liquids of varying composition. The effect is commonly observed in flow injection analysis when the samples which are injected into a non-segmented reagent stream differ in composition from that stream $[9,10]$. According to Dias et al. [9], the refractive index gradients act as "liquid lenses as well as a myriad of randomly distributed transient mirrors". In FIA flowcells, these effects can deviate the detector light beam so that the sharp change of the signal generally prevents correct measurement of the sample absorbance. The effect can be reduced with a flowcell design that measures light absorbance across or at an angle to the direction of flow or which reflects the light so that measurement takes place in both directions along the flow [18 - 20].

In segmented-flow seawater analysis a similar effect occurs when the flow is debubbled before entering the flowcell and the sample and the inter-sample wash have different salinity. In such a case the refractive index of the liquid in the flowcell changes over a period of few seconds, within a transitional mixing zone at the beginning and end of each sample where the sample and wash are both present in the cell. Laminar flow results in the faster-moving liquid in the centre of the flowcell generating an inconsistent gradient of refractive index which distorts the light beam, producing apparent absorbance variations, which may be positive and/or negative. The signal shape is unpredictable since it depends on the refractive index of the sample and the wash liquid and whether the laminar flow tends to form a lens which is convergent or divergent. However, in SFA the transition zone is relatively short in comparison to the steady-state plateau generated by a sample peak so that it does not usually prevent accurate measurement. Nevertheless, this effect may slow down the analytical throughput and it should be minimised as far as possible. 
Some oceanographers using SFA instruments with debubbled flowcells have considered that analysing seawater samples with fresh water as the wash liquid produced a schlieren effect too large to enable high throughput with accurate results. To overcome the problem they used artificial seawater (ASW) or low nutrient seawater (LNSW) for the baseline (Gordon, [11]). Although convenient to minimise schlieren effects for the analysis of oceanic samples whose salinity is almost constant, this alternative is not applicable to coastal and estuarine samples with salinity varying over a large range. Additionally, in the analysis of oceanic samples the necessary determination of the nutrient concentration in the baseline water is relatively tedious and liable to introduce additional errors if not accurately performed. Using water as the baseline solution has the undoubted advantages of being simpler and readily accessible and of providing a true "zero absorbance" reference level, so we recommend this option and describe in section 3.8.2 how the dynamic influence can be minimized by using a suitable analyzer configuration.

\subsection{Salinity effects on the reaction rate or end point}

Among the nutrients usually measured in natural waters (nitrate, nitrite, silicate, phosphate and ammonium), the most commonly used methods for silicate and ammonium determinations are significantly affected by salinity.

Silicate determination provides an example for the comparison of methods where the formation of the same end product (a silico-molybdate complex then reduced to obtain an intense blue colour) under different analytical conditions leads to a wide range of salt effects. Method data from the literature are compared in Table 2, from which it can be seen that salt effects up to $16 \%$ have been reported. All workers found a linear salt effect, which is easy to express and correct. As shown in the table, the effect is partly a function of the dilution ratio of the sample in the reaction medium, but other reaction conditions influence its magnitude. For example, when an original manual method [14] was automated [4], the salt effect was reduced to about half its initial value. It is clear that analysts should not apply salt effect values directly from literature data, but should determine their own values in their specific laboratory conditions.

Of the nutrient methods, ammonium determination generally shows the greatest and most complex influence from varying salinity. Two commonly used types of method are the colorimetric determination with alkaline phenate and the fluorometric determination with orthophthalaldehyde (OPA). Table 4 compares the maximum salt effect of four methods based on these two principles over the salinity range 0-35. Due to non-linearity, the maximum occurs between a salinity of 5 and 15 .

To illustrate how the salt effect varies as a function of salinity, we compared one method based on each principle [13 and 15], and the results are shown in Figure 1. The phenate method shows the greater influence from salinity and in both methods the non-linear relationship between sensitivity and salinity makes correction tedious.

Of the other nutrients analysed with currently used methods, phosphate does not exhibit a salt effect, according to Murphy and Riley [17] and for nitrate and nitrite the salt effect is usually negligible. It should be noted that an automated method may exhibit a salt effect while the corresponding manual version is free of it: this can be due to kinetic factors or differential adsorption onto the manifold walls as a function of salinity. 


\subsection{General case for measuring and correcting the salt effect}

The influence of salinity on method results is quantified by measuring equi-molar standard solutions of the analyte made up in a series of dilutions of a basic matrix (preferably natural low nutrient seawater) from lowest to highest expected sample salinity.

Salt effect correction may appear to be a simple concept but can give rise to erroneous results if incorrectly applied. Hansen and Koroleff [5] proposed a two-step procedure starting with the measurement of a range of standards in waters covering a range of salinities. They then calculated the relative deviations and plotted them vs. salinity to obtain a slope used in a correction formula.

Here, we propose a one-step procedure. Standards of the same concentration (in the middle of the usual range) are prepared in a series of waters of different salinities. Because there may be a non-linear relationship between sensitivity and salinity at least five dilutions should be used. The results are computed as relative apparent concentrations, the value of 1.00 being attributed to the concentration obtained at a reference salinity (usually the highest or the lowest one). The data are plotted versus salinity (see example for ammonium in Figure 1) so that the relative salt effect ( $r s e)$ can be determined at any intermediate salinity. If calibration is performed at the reference salinity, sample measurements at salinity $S$ are corrected by dividing them by the factor $(1+r s e)$ determined at salinity $S$ to obtain the true concentration. If calibration is performed at a salinity different from the reference salinity, rse can easily be recomputed from the initial salt effect data to obtain the salt effect relative to the actual calibration salinity. We verified that the salinity effect is independent of analyte concentration, in confirmation of the results of Hansen and Koroleff [5].

\subsection{Correction method for linear salt effect with samples or standards of any salinity}

When the salt effect is a linear function of salinity, the slope " $A$ " is determined. Generally, apparent concentrations are lower in sea than in fresh water, in which case $A$ is negative.

Then, in a normal run with a calibration performed at a salinity of $S_{\text {Calib }}$ and an uncorrected concentration $C_{\text {uncor }}$ for a sample at a salinity of $S_{\text {Sample }}$, the relationship to obtain the corrected concentration $C_{c o r}$ is:

$\mathrm{C}_{\text {cor }}=\mathrm{C}_{\text {uncor }} /\left[1+\mathrm{A} \times\left(\mathrm{S}_{\text {Sample }}-\mathrm{S}_{\text {Calib }}\right)\right]$

Once A has been determined, this correction can be applied automatically if the analyzer measures the salinity or has provision for it to be input.

Where the salt effect is non linear, a more complex correction can be applied by using a polynomial equation derived from deviations from expected concentrations, or each sample's apparent concentration can be individually corrected according to the relative salt effect factor obtained from the plot versus salinity.

\subsection{A simplified correction method for linear and non-linear salt effects where the salinity of standards is constrained}

A universal simplified method can be used to correct the salt effect under only one constraint: the salinity of the standards should be equal to the reference salinity where the value for the relative concentration is defined as 1.00 . The full salt effect function $F(S)$, whether linear or not, is determined from the same plot as above and the corrected concentration is:

$$
\mathrm{C}_{\mathrm{cor}}=\mathrm{C}_{\mathrm{uncor}} / \mathrm{F}(\mathrm{S})
$$


With this method, a difference of $\Delta S= \pm 1$ between the calibration salinity and the reference salinity generates an error of only $0.3 \%$ on the corrected concentration for a salt effect as high as $10 \%$, as shown in the following example where the reference salinity is 35 and a sample in pure water shows a salinity effect of $10 \%$. If the calibration standards have a salinity of 34 instead of 35 the difference in the salinity effect is $1 / 35$ or approx. 0.03 . The relative error on a salt water sample is thus $0.1 \times 0.03=0.3 \%$. Using a method with a salt of effect of $5 \%$, it is $0.15 \%$.

\subsection{Reducing the salt effect}

Methods free of salt effects are desired to simplify analyses, minimise corrections and improve the accuracy of results. Where this is not possible, minimizing the salt effect should be the objective. If the salt effect makes computation of results tedious, analysts may be able to change their method for one having a lesser and/or linear effect. It should be noted that using a more sensitive method enables the nutrient to be measured at lower concentrations, so that by increasing the sample dilution in the manifold the salt effect is reduced in proportion to the dilution factor.

\subsubsection{Static matrix effects on the optical signal: RIB}

RIB is proportional to salinity. It can be quantified according to [2] by setting up the SFA method, establishing a baseline using pure water as the sampler wash and replacing one reagent with another which omits a vital colour-producing chemical. Seawater samples are then passed through the system to measure the RIB. The sampling time should be long enough to establish a steady-state peak plateau during which the concentration does not change with time. For the most commonly used methods, we recommend omitting molybdate for silicate and phosphate measurements, the chlorine donor for the phenate ammonium method, and naphthylethylenediamine dihydrochloride for nitrate and nitrite. Under these conditions the detector output from the sample peaks, the so-called RIB, actually represents the sum of refractive index (the major contribution for most systems) and colour and turbidity contributions if any. At a salinity of 35 the RIB is about $0.5 \mu \mathrm{mol} / \mathrm{L}$ with silicate method [13] (AAII) and $0.05 \mu \mathrm{mol} / \mathrm{L}$ with method [12] (AA3). It is about $0.2 \mu \mathrm{mol} / \mathrm{L}$ with the colorimetric phenate method [13] for ammonia, but the fluorometric OPA determination described in [15] does not exhibit an RIB effect and therefore such a correction is not necessary when this method is used; however, that should be checked if using a different fluorometer. Figure 2 shows the RIB peak for a seawater sample measured on an analyzer for phosphate using three different detectors under identical chemical conditions. This illustrates how the RIB is influenced by the detector design. The detector used for the first measurement uses a debubbled tubular flowcell with rounded ends and a large tungsten filament lamp (RIB 0.12 $\mu \mathrm{mol} \mathrm{L}^{-1}$ ). The second uses a cylindrical flowcell with parallel ends, fibre optics and a small krypton-filled tungsten filament lamp; the bubbles pass through the flowcell and software in the detector eliminates their interfering effect $\left(\mathrm{RIB} \sim 0.10 \mu \mathrm{mol} \mathrm{L}^{-1}\right)$. The third detector is similar to the second but the optics have been modified to reduce the aperture and create a more nearly parallel light beam (RIB - $0.02 \mu \mathrm{mol} \mathrm{L}^{-1}$ ).

"Matrix photometers" which pass white light through the flowcell and then split it, measuring one beam at the chromophore lambda max and another at a wavelength that is not absorbed by the chromophore, were not used in this work because they are not compatible with an LED light source, which we prefer for photometers to be used in a ship-board laboratory as they are unaffected by vibration. We have also found that bichromatic photometers have a tendency to drift as the spectrum from a tungsten lamp changes with temperature, and prefer a dual-beam same-wavelength type for high sensitivity work. 


\subsubsection{Dynamic salt effects on the optical signal}

If air segmentation is maintained within the flowcell, direct mixing between the sample and wash liquid is effectively eliminated. Schlieren effects at the beginning and end of sample peaks are greatly reduced because there is no direct interface between the samples and wash solution. This is particularly beneficial when the sample and wash have different refractive indices.

The effect of "bubble-through" operation is illustrated in Figure 3 which shows SFA analyzer peaks from seawater samples interspersed with pure water wash which were passed through a $10 \mathrm{~mm}$ bubble-through flowcell and then into a second $50 \mathrm{~mm}$ flowcell with a debubbler. These are the flowcell types commonly used on AA3 and AAII analyzers respectively for seawater analysis, and some analysts prefer a long flowcell to reduce the influence of electronic noise and drift, which can be significant with some detectors. The same solution is being measured in both cases, and the gain on the display was set such that equal concentration standards show approximately the same height with each flowcell. Note that the deeper dips between the peaks on the left-hand $(10 \mathrm{~mm}$ cell) trace reflect the lower dispersion in the bubble-through flowcell and are not representative of sensitivity. No signal averaging was used for either measurement. The time constant of the detector amplifier and the A/D converter period are insignificant in comparison to the 2-second period between liquid segments arriving at the flowcell. In the bubble-through flowcell the separation between the peaks is more well-defined due to the lower dispersion within the cell, and the steady-state peak plateau where the concentration of the sample in the flowcell is at its maximum value is about $50 \%$ longer. The extended steady-state time may be used to improve the precision of measurement or the plateau can be shortened to the time generated by the debubbled flowcell so that the analysis rate is increased. If the analyzer does not support bubble-through operation, adverse effects from the spurious peaks or spikes at the beginning of the peaks from the debubbled flowcell can be reduced by ensuring that the sampling time is long enough to generate a steady-state peak plateau and adjusting the peak picker start and stop time so that only the steady-state part of the peak is searched when calculating the peak result. This type of dynamic effect is practically independent of chemistry. Its magnitude and period depend on the difference in RI between sample and wash, the flowcell length and internal diameter, and the flow rate through the cell.

Even with a bubble-through flowcell, some disturbance may be seen at the beginning or end of the peaks when working at high sensitivity due to transfer of the surface layer inside the flowcell as the bubble passes through, but the magnitude is small and the effect is of short duration so that the peak picker can easily be set to ignore it.

\subsection{Measuring and correcting sample colour and refractive index blank}

Due to the relative ease of obtaining nutrient-free deionised water as opposed to nutrient-free seawater, we recommend the following procedure.

1. Use ultra-pure water for the sampler wash and therefore the baseline.

2. Prepare calibration standards in low-nutrient seawater (LNSW). It is not necessary to know the value of the residual LNSW signal (= nutrient concentration + RIB) because it will be corrected automatically when using this procedure.

3. Set up a run with standards and samples as normal. Include a zero standard of LNSW in the calibration set and do not include the baseline in the calibration. After the last sample insert a sufficient number of multiple baseline periods taken from the sampler wash receptacle so that the time taken to sample them is equal to about 5 minutes 
longer than the time taken for a sample to reach the detector. On an analyzer running at 30 samples per hour about 7 or 8 sample periods are normally sufficient. Follow these by repeats of the samples for which it is desired to measure and correct the blank value. Set the software to calculate the sample results using only the slope of the calibration curve and ignoring the intercept. By doing this the residual nutrient concentration and RIB of the LNSW standards are eliminated from the calculations.

4. Start the run.

5. After the first of the multiple baseline periods has reached the detector, replace one of the reagents with its "RIB reagent" equivalent which omits the colour-producing chemical as described previously.

6. A new baseline will be established using the "RIB reagent", after which the samples are measured again. This time the sample peaks are the Rlbs.

7. Once the uncorrected sample results and Rlbs have been computed, subtract the sample RIB values to obtain the true nutrient concentrations.

In practice when analysing a series of similar samples such as open ocean water it is only necessary to analyse a few samples to obtain the blank value as it does not differ significantly from one sample to another.

Figure 4 shows the changeover between the normal and the blank-correction sections of the run in diagramatic form, and Figure 5 shows the actual chart from a short run of a series of replicate low samples.

\section{Conclusion}

This paper reviews and summarizes all the matrix effects encountered in the determination of nutrients in saline waters using SFA, previously available only in several independent papers. Due to improvements in analyzer performance and calibration reference standards the accuracy of measurement becomes more sensitive to matrix errors, making it appropriate to re-examine these effects in the context of modern instrument design.

We suggest that analysts examine the influence of salinity on their methods, compare it to that of other methods and consider whether the chemicals or their concentrations can be modified to significantly minimise the influence of salinity, or if an alternative method can be used. For correction of the chemical salt effect we propose a simple procedure which should make handling data less tedious, thus reducing computation errors and improving data quality.

Hardware-specific dynamic effects due to refractive index changes which affect peak reading are greatly reduced by improved instrument optics and by avoiding flowcell debubbling so that they can be eliminated as a source of error while still maintaining an acceptable sampling rate.

As a consequence of reduced refractive index effects, we are in favour of using pure water for the baseline instead of saline water (ASW or LNSW): this has the advantages of being simpler, readily accessible and of providing a true "zero absorbance" reference level. Measurement of static RIB is quite easy on a few representative samples, correction is simply applied, and the measurement and correction procedures are capable of being automated. 
Thanks to Divya Singh of Technische Universität Hamburg-Harburg for help with literature searches and laboratory work.

\section{References}

[1] M. Aoyama, J. Barwell-Clarke, S. Becker, M. Blum, E. S. Braga, S. C. Coverly, E. Czobik et al., Technical Reports of the Meteorological Research Institute, Tsukuba, Japan, No. 58, 2008. Available at http://www.mri-jma.go.jp/Publish/Technical/index_en.html

[2] T. C. Loder, P. M. Glibert, Advances in automated analysis; Technicon International Congress 1976: (proceedings) - v. 2: Industrial symposia, pages 48-56.

Conference: Technicon Int. Congr. 7. New York, N.Y. (USA), 13-15 Dec 1976. .Publ. place: Tarrytown, N.Y. (USA). Publisher: Mediad Inc. Publ. date: 1977. Inclusive pages: 366 . Also available as P.M. Glibert and T.C. Loder, "Automated analysis of nutrients in seawater: A manual of techniques, Technical Report WHOI-77-47, Woods Hole Oceanographic Institution (1977), p. 46, available on-line as Appendix A of "Final Data Report for Analysis of Water Quality Samples Taken During a New York Bight Survey in July 1988" at nepis.epa.gov/Exe/ZyPURL.cgi?Dockey=20005289.txt

[3] S. C. Pai, G. C. Gong, K. K. Liu, Anal. Chim. Acta, 434 (2001), 209-216.

[4] P.G. Brewer, J.P. Riley, Anal. Chim. Acta 35 (1966) 514-519.

[5] H.P. Hansen, F. Koroleff, in: K. Grasshoff, K. Kremling, M. Ehrhardt (Eds) Methods of seawater analysis, third ed., Wiley-VCH, Weinheim, 1999, 159-228.

[6] P. N. Froelich, M. E. Q. Pilson, Wat. Res 12 (1978) 599-603.

[7] A. Aminot, R. Kérouel, Dosage automatique des nutriments dans les eaux marines, Quae, Paris, 2007.

[8] R. Hooke, Micrographia, The Royal Society, London, (1665) 217-219

[9] A. C. B. Dias, E. P. Borges, E A. G. Zagatto, P. J. Worsfold, Talanta (2006) 1076- 1082

[10] I. D. McKelvie, D. M.W. Peat, G. P. Matthews, P. J. Worsfold, Anal Chim Acta 351 (1997) 265-271.

[11] L. I. Gordon, J. C. Jennings, Jr., A. A. Ross, J. M. Krest, WOCE Hydrographic Program Office, Method Manual WHPO 91-1, 1993; and http://cchdo.ucsd.edu/manuals/pdf/91_1/gordnut.pdf

[12] A. Aminot, R. Kérouel, S. C. Coverly, in: O. Wurl (Ed), Practical Guidelines for the Analysis of Seawater, Inc. Boca Raton, 2009, pp. 143-178.

[13] P. Tréguer, P. Le Corre, Manuel d'analyse des sels nutritifs dans l'eau de mer, Université de Bretagne Occidentale, Brest (France), 1975.

[14] J. B. Mullin, J. P. Riley, Anal. Chim. Acta 12 (1955) 162-176.

[15] R. Kérouel, A. Aminot, Mar. Chem. 57 (1997) 265-275.

[16] R. F. C. Mantoura, E. M. S Woodward, Est. Coast. Shelf Sci. 17 (1983) 219-224.

[17] J. Murphy, J. P. Riley, Anal. Chim. Acta 27 (1962) 31-36.

[18] S. Jambunathan, P. K. Dasgupta, D .K. Wolcott, G. D. Marshall, D. C. Olson, Talanta 50 (1999) 481-490.

[19] P. S. Ellis, A. J. Lyddy-Meaney, P. J. Worsfold, I. D. McKelvie, Anal. Chim. Acta 499 (2003) 81-89.

[20] S. K. Mishra, P. K. Dasgupta, Anal. Chim. Acta 605 (2007) 166-174. 


\section{Tables}

Table 1. Summary of methods used in experimental work

\begin{tabular}{|c|c|c|c|c|c|c|}
\hline \multirow[t]{2}{*}{ Nutrient } & \multirow[t]{2}{*}{ Reference } & \multicolumn{3}{|c|}{ Working reagents } & \multicolumn{2}{|c|}{ Flow $\left(\mathrm{mL} \mathrm{min}^{-1}\right)$} \\
\hline & & $R_{n}$ & Compounds & $\mathrm{g} \mathrm{L}^{-1}$ & Reagents & Sample \\
\hline \multirow{3}{*}{$\begin{array}{l}\text { Ammonium } \\
\text { (fluorometry) }\end{array}$} & [12] and [15] & $R$ & $\mathrm{Na}_{2} \mathrm{~B}_{4} \mathrm{O}_{7}, 10 \mathrm{H}_{2} \mathrm{O}$ & 30 & 0.80 & 1.00 \\
\hline & & & Orthophthalaldehyde & 0.8 & & \\
\hline & & & $\mathrm{Na}_{2} \mathrm{SO}_{3}$ & 0.016 & & \\
\hline \multirow{6}{*}{$\begin{array}{l}\text { Ammonium } \\
\text { (colorimetry) }\end{array}$} & [7] & $R_{1}$ & Trisodium citrate, $2 \mathrm{H}_{2} \mathrm{O}$ & 200 & 0.10 & 1.77 \\
\hline & . & & & & & \\
\hline & & & $\begin{array}{l}\text { Sodium nitroprusside, } \\
2 \mathrm{H}_{2} \mathrm{O}\end{array}$ & 1.6 & & \\
\hline & & $R_{2}$ & Phenol & 17.6 & 0.10 & \\
\hline & & $R_{3}$ & $\mathrm{NaOH}$ & 11 & 0.10 & \\
\hline & & & Na-dichloroisocyanurate & 2.0 & & \\
\hline \multirow[t]{4}{*}{ Phosphate } & [12] and [15] & $R_{1}$ & Ascorbic acid & 4 & 0.80 & 1.77 \\
\hline & & $R_{2}$ & Sulphuric acid (mL L-1) & $1.22^{\mathrm{a}}$ & 0.42 & \\
\hline & & & $\begin{array}{l}\text { Ammonium Molybdate, } \\
6 \mathrm{H}_{2} \mathrm{O}\end{array}$ & 6 & & \\
\hline & & & $\begin{array}{l}\mathrm{KSb}(\mathrm{III}) \text { oxytartrate, } \\
0.5 \mathrm{H}_{2} \mathrm{O}\end{array}$ & 0.15 & & \\
\hline
\end{tabular}

a $\mathrm{mol} \mathrm{L}^{-1}$. 
Table 2. Maximum salt effect in the determination of silicate over a 0-35 salinity range, according to various workers.

\begin{tabular}{|c|c|c|c|c|}
\hline Reference & $\begin{array}{l}M<!-- \text { no-mfc } \\
-\rightarrow / A<!--/ \text { no-mfc } \\
\rightarrow \rightarrow a\end{array}$ & $\begin{array}{l}\text { Reducing } \\
\text { agent }\end{array}$ & $\begin{array}{l}\text { Sample in } \\
\text { reaction medium } \\
(\%)\end{array}$ & $\begin{array}{l}\text { Salt effect (\%) over } \\
\text { a 0-35 salinity range }\end{array}$ \\
\hline $\begin{array}{l}\text { Mullin and Riley } \\
\text { [14] }\end{array}$ & $\mathrm{M}$ & Metol sulfite & 30 & 10 \\
\hline $\begin{array}{l}\text { Brewer and } \\
\text { Riley [4] }\end{array}$ & A & Metol sulfite & 35 & 5.4 \\
\hline $\begin{array}{l}\text { Hansen and } \\
\text { Koroleff [5] }\end{array}$ & $\mathrm{M}$ & $\begin{array}{l}\text { ascorbic } \\
\text { acid }\end{array}$ & 91 & 16 \\
\hline $\begin{array}{l}\text { Tréguer and Le } \\
\text { Corre [13] }\end{array}$ & A & Metol sulfite & 45 & 7.9 \\
\hline $\begin{array}{l}\text { Aminot et al. } \\
\text { [12] }\end{array}$ & A & $\begin{array}{l}\text { ascorbic } \\
\text { acid }\end{array}$ & 10 & 4.0 \\
\hline
\end{tabular}

Table 3. Maximum salt effect in the determination of ammonium over a 0-35 salinity range, according to various workers.

\begin{tabular}{|c|c|c|c|}
\hline Reference & $\begin{array}{l}\mathrm{M}<!-- \text { no-mfc } \rightarrow / A<!--/ \text { no-mfc } \\
\rightarrow \rightarrow\end{array}$ & Method type & $\begin{array}{l}\text { Salt effect } \\
(\%)\end{array}$ \\
\hline Tréguer and Le Corre [13] & A & $\begin{array}{l}\text { Phenate } \\
\text { colorimetry }\end{array}$ & 9.1 \\
\hline $\begin{array}{l}\text { Mantoura and Woodward } \\
\text { [16] }\end{array}$ & A & $\begin{array}{l}\text { Phenate } \\
\text { colorimetry }\end{array}$ & $\sim 8$ \\
\hline Kérouel and Aminot [15] & A & OPA fluorometry & 3.1 \\
\hline Hansen and Koroleff [5] & $\begin{array}{l}\mathrm{M}<!-- \text { no-mfc }-->/ A<!--/ \text { no-mfc } \\
-\rightarrow\end{array}$ & $\begin{array}{l}\text { Phenate } \\
\text { colorimetry }\end{array}$ & 26 \\
\hline
\end{tabular}


Figure 1. Salinity influence on two different determinations for ammonium, relative to calibration in seawater. $\bullet$ : phenate colorimetry; $\bigcirc$ : OPA fluorometry

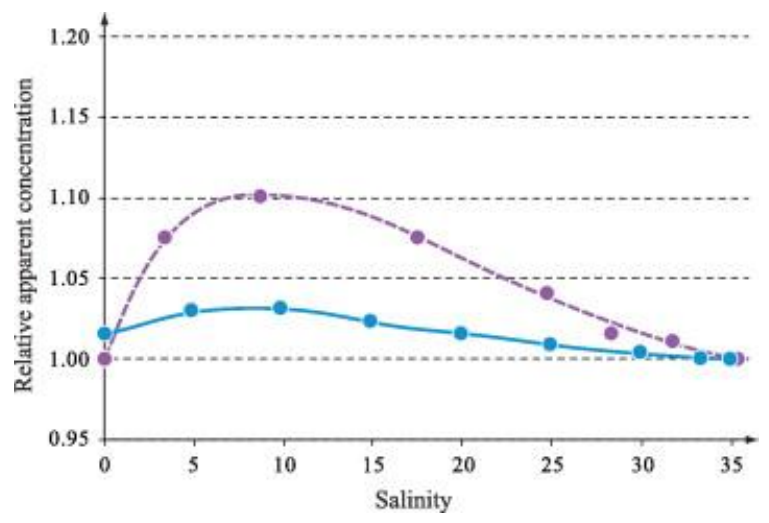

Figure 2. RIB peaks generated by different detectors. Sea water samples interspersed with pure water wash in a phosphate analysis manifold, using "refractive index blank" reagents which omit the molybdate so as to show only refractive index effects. The scale is in micromoles per litre. The time scales are not identical.

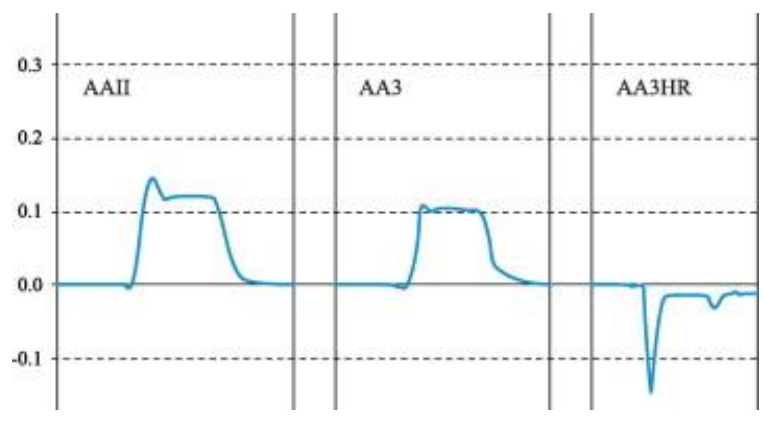

Figure 3. Flowcell influence on peaks from seawater samples interspersed with pure water wash. Left: a $10 \mathrm{~mm}$ bubble-through flowcell. Right: a $50 \mathrm{~mm}$ flowcell with debubbler.

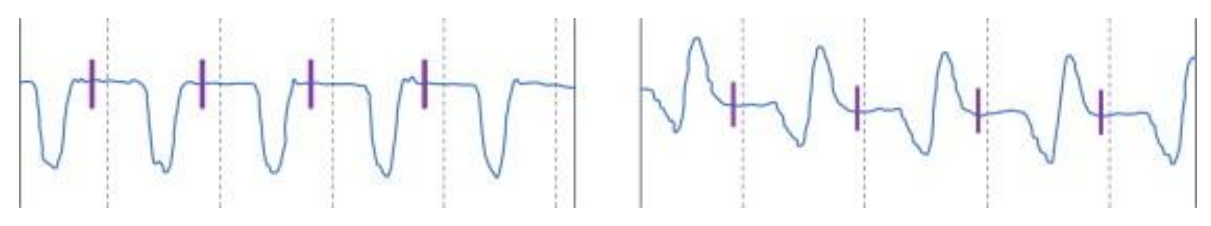


Figure 4. Changeover between normal and refractive index blank (RIB) determination in a blank-correction run for samples of variable salinity. The system runs with a pure water baseline. The baseline signal difference between normal and RIB reagents corresponds to the reagent blank.

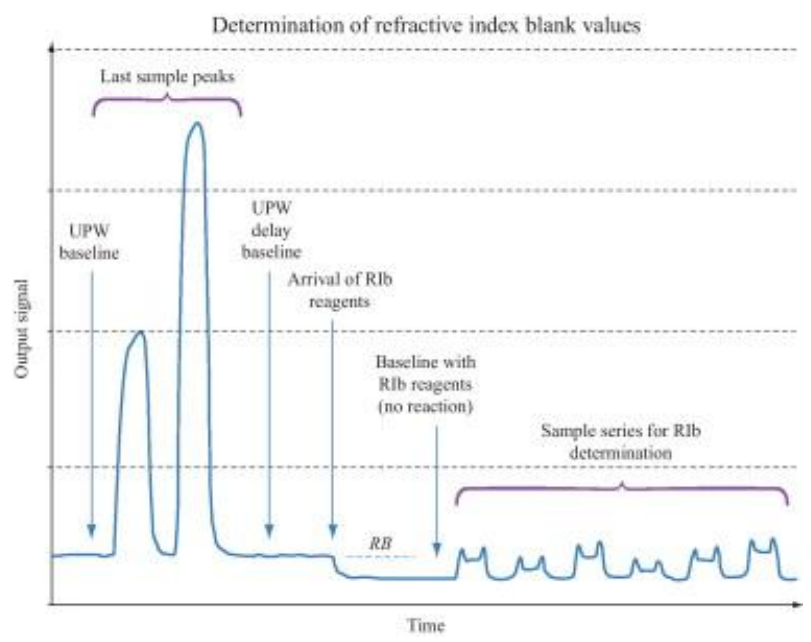

Figure 5. Chart from a blank-correction run for phosphate on a series of samples with low concentration. The reagent changeover occurs at about 30 minutes. Full scale is equivalent to about $6 \mu \mathrm{M}$. In this case the RIB peak values are negative.

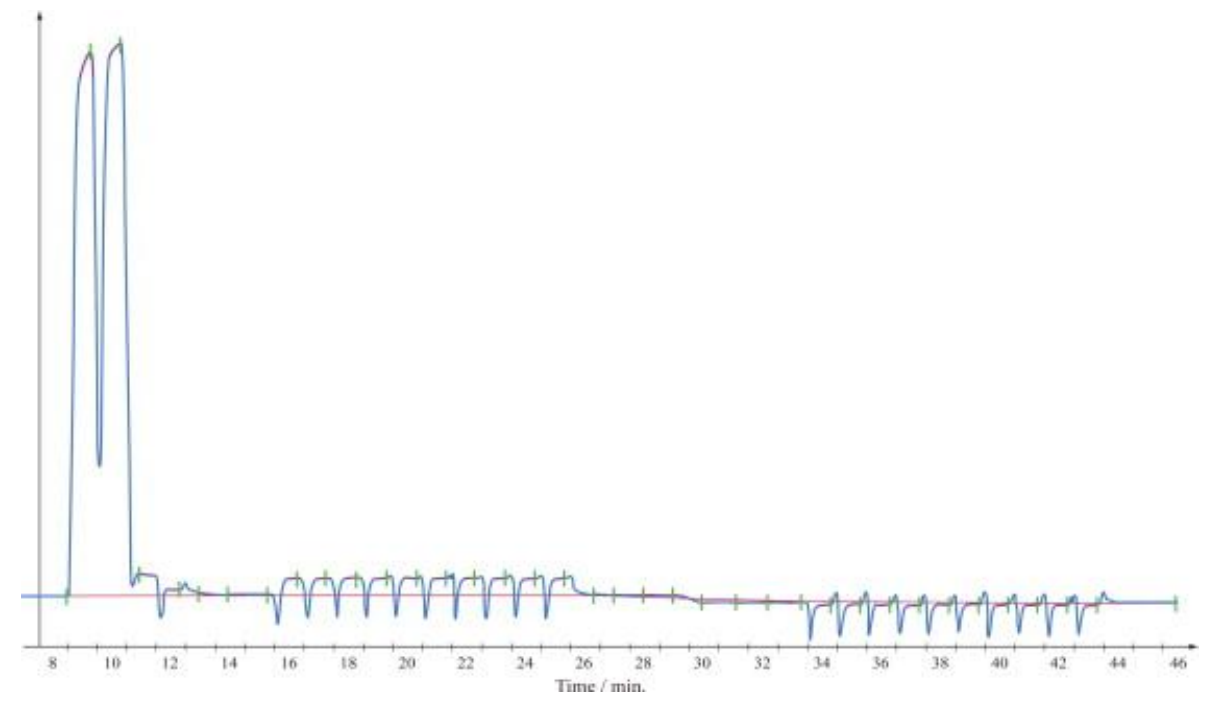

